\title{
Metastable Nonextremal Antibranes
}

\author{
J. Armas, ${ }^{1,2}$ N. Nguyen, ${ }^{3}$ V. Niarchos, ${ }^{3}$ N. A. Obers, ${ }^{4}$ and T. Van Riet ${ }^{5}$ \\ ${ }^{1}$ Institute for Theoretical Physics, UVA, 1090 GL Amsterdam, Netherlands \\ ${ }^{2}$ Dutch Institute for Emergent Phenomena, Netherlands \\ ${ }^{3}$ Department of Mathematical Sciences and Centre for Particle Theory Durham University, Durham DH1 3LE, United Kingdom \\ ${ }^{4}$ The Niels Bohr Institute, University of Copenhagen Blegdamsvej 17, DK-2100 Copenhagen $\varnothing$, Denmark \\ ${ }^{5}$ Instituut voor Theoretische Fysica, K.U.Leuven, Celestijnenlaan 200D, B-3001 Leuven, Belgium
}

(Received 17 December 2018; published 9 May 2019)

\begin{abstract}
We find new and compelling evidence for the metastability of supersymmetry-breaking states in holographic backgrounds whose consistency has been the source of ongoing disagreements in the literature. As a concrete example, we analyze anti-D3 branes at the tip of the Klebanov-Strassler throat. Using the blackfold formalism we examine how temperature affects the conjectured metastable state and determine whether and how the existing extremal results generalize when going beyond extremality. In the extremal limit we exactly recover the results of Kachru, Pearson, and Verlinde, in a regime of parameter space that was previously inaccessible. Away from extremality we uncover a metastable black NeveuSchwarz five-brane (NS5) state that disappears near a geometric transition where black anti-D3 branes and black NS5 branes become indistinguishable. This is remarkably consistent with complementary earlier results based on the analysis of regularity conditions of backreacted solutions. We therefore provide highly nontrivial evidence for the metastability of antibranes in noncompact throat geometries since we find a consistent picture over different regimes in parameter space.
\end{abstract}

DOI: 10.1103/PhysRevLett.122.181601

Introduction.-An understanding of controlled supersymmetry (SUSY) breaking in string theory is arguably one of the most important goals in order to progress string phenomenology. Thanks to holography, the use of controlled SUSY breaking in string backgrounds would improve our understanding of strongly coupled phenomena in field theories with broken SUSY. The latter is an important issue independent of the conjecture that string theory is the quantum theory underlying our physical world.

One of the canonical methods for SUSY breaking in string theory employs antibranes in warped throats. The study of this mechanism has a long history, starting with the original papers $[1,2]$ whose motivation was the holographic description of dynamical breaking of supersymmetry. Since then, antibranes have become an indispensable tool to break SUSY in various contexts. The applications beyond holography include de Sitter [3], inflationary model building [4], and the construction of nonextremal black hole microstates [5]. For some of these applications additional complications due to the compactness of extra dimensions arise, which we will not address. Instead, we restrict to

Published by the American Physical Society under the terms of the Creative Commons Attribution 4.0 International license. Further distribution of this work must maintain attribution to the author(s) and the published article's title, journal citation, and DOI. Funded by SCOAP ${ }^{3}$. the study of antibranes in the noncompact KS throat. The motivation for that is twofold. On one hand, the KS throat is a prime example of a top-down holographic background with broken conformal symmetries that exhibits confinement. Studying SUSY breaking in this context is therefore a concrete example of the ultimate goal to get a computational handle on QCD-like field theories in the absence of SUSY. On the other hand, for string phenomenology it has been argued that the KS throat at least constitutes a local description of the more elusive compact throat geometries.

In this Letter we present entirely novel arguments for the highly debated metastability of SUSY-breaking states in these throats and, at the same time, demonstrate how to extend the analysis to situations that incorporate finite temperature effects in the holographic dual, offering new methods to study phase transitions in this context.

Our candidate metastable states are described by $\overline{D 3}$ 's at the tip of the throat. They can decay because the surrounding 3-form fluxes induce delocalized $D 3$ charges out of which $D 3$ 's can nucleate and annihilate with the $\overline{D 3}$ 's. This process can be described in terms of brane polarization in which the $\overline{D 3}$ 's "puff" into a spherical NS5 wrapping a contractible $S^{2}$ inside the $S^{3}$ cycle [2]. As the NS5 moves over the $S^{3}$, it changes the sign of the $D 3$ charges it carries, effectively mediating the brane-flux decay. To find a metastable state the NS5 needs to find a balance between the $\mathrm{H}_{3}$-flux force that wants to push the NS5 over the $S^{3}$ 
and the force of its own "weight" doing the opposite. In the probe limit, KPV found that such a balance of forces exists whenever the ratio $p / M$ is small enough [2]. Here, $p$ denotes the number of $\overline{D 3}$ 's and $M$ the quantum of 3-form flux piercing the $S^{3}$.

The existence of this KPV state has been refuted in various works starting with the investigations of Ref. [6]. The problem, found at the time, arises when trying to go beyond the probe limit and investigate what happens once the branes backreact. In particular Ref. [6], and many subsequent works [7], found that the backreacted geometry had singular 3-form fluxes in such a way that it would cause immediate brane-flux decay [8]. As a response, Ref. [9] argued that the singularity is renormalized in such a way that does not affect stability when $p=1$, which is a case that is not amenable to a supergravity analysis. References [10,11] argued that metastability can also be retained when $p \gg 1$ since the observed singularities cannot be proven to exist once one backreacts spherical NS5's instead of pointlike $\overline{D 3}$ 's. In fact, all proofs of unphysical singularities rested on an assumption which was in contradiction with $\mathrm{KPV}$ from the start, since $\overline{D 3}$ metastable states are really NS5 states.

Several studies [12-15] have investigated the effect of adding temperature to the $\overline{D 3}$ 's. Most of these works were motivated by the would-be singularity in the 3 -form fluxes. Whether or not a singularity can be cloaked by a horizon that arises when moving away from extremality is believed to be an important criterion for deciding the fate of singularities [16]. Although strong indications were found that one should not worry about singularities at all [9-11], it remains an outstanding problem to understand what happens when the $\overline{D 3}$ 's are at finite temperature. If the state would destabilize infinitesimally away from extremality, it would be a sign of being gapless, which is not wanted. In this Letter we present additional evidence that this does not happen and provide new, previously inaccessible, quantitative results that support the picture of Refs. [10,11].

Our approach.-From the NS5 viewpoint D3-NS5 polarization is most naturally described in the supergravity regime where $g_{s} p \gg 1$ (see, e.g., Ref. [17]). This involves a daunting task: solving the type IIB supergravity equations to find D3-NS5 bound state solutions wrapping an $S^{2}$ in the presence of the fluxes of the KS background. In this Letter, we attack this problem using blackfold techniques [18-20].

In the blackfold formalism, problems of the above type are treated systematically by setting up a scheme of matched asymptotic expansions, where the solution is approximated in a far zone by the background solution of interest (here the KS background) and in a near zone by a uniform flat-space $p$-brane solution (here the D3-NS5 bound state). This scheme is possible when the characteristic length scales of the near-zone solution, denoted collectively by $r_{b}$, are hierarchically smaller than the characteristic length scales $\mathcal{R}$ of world volume inhomogeneities, and the characteristic length scales $L$ of the background. The regime $r_{b} \ll \mathcal{R}, L$ is a regime of long-wavelength expansions. For a general discussion of blackfolds and the approximations they entail we refer the reader to Ref. [20] and references therein. The specifics of the regimes of interest in our context are summarized below.

A key component of the above analysis reformulates part of the supergravity equations as an effective world volume theory. In the case at hand, this is a supergravity-derived $6 d$ effective theory that describes the long-wavelength properties of NS5 branes with dissolved 3-brane charge. In what follows, we focus exclusively on the leading-order equations of the $6 d$ theory (blackfold equations). At the very least, these equations pose necessary conditions for the existence of the long-sought supergravity solution in the above regime.

Forced blackfold equations.-The leading order blackfold equations for black branes in the presence of general external fluxes in (super)gravity were obtained in Ref. [20]. We consider $\overline{D 3}$-NS5 branes at the tip of the KS throat, where in appropriate units the string frame metric is [21]

$d s^{2}=g_{s} M b_{0}^{2} \ell_{s}^{2}\left[d s^{2}\left(\mathbb{M}_{0123}\right)+d \Omega_{3}^{2}+d r^{2}+r^{2} d \Omega_{2}^{2}\right]$.

Here, $\mathbb{M}_{0123}$ is Minkowski space in the directions 0123, $d \Omega_{n}^{2}$ is the metric element of the round unit $n$ sphere, $b_{0}^{2} \simeq 0.93266$, and $\ell_{s}$ the string scale. There is also a Ramond-Ramond (RR) field strength $F_{3}=d C_{2}=$ $2 M \ell_{s}^{3} \operatorname{Vol}\left(S^{3}\right)$ across the $S^{3}$ and a 7-form flux $H_{7}=d B_{6}$ dual to $H_{3}=d B_{2}$,

$H_{7}=\frac{1}{g_{s}^{2}} \star H_{3}=-\frac{\ell_{s}^{4}}{g_{s}} d x^{0} \wedge d x^{1} \wedge d x^{2} \wedge d x^{3} \wedge F_{3}$.

The dilaton is constant and the self-dual 5-form field strength vanishes at the tip.

The boosted D3-NS5 bound state solution in flat space, which forms the seed of our blackfold expansion, is well known (see, e.g., Refs. [22,23]). The following thermodynamic data of this solution (in the Einstein frame) are needed for our purposes: the energy-momentum tensor

$$
\begin{aligned}
T_{a b}= & \mathcal{C}\left[r_{0}^{2}\left(u_{a} u_{b}-\frac{1}{2} \gamma_{a b}\right)-r_{0}^{2} \sin ^{2} \theta \sinh ^{2} \alpha \hat{h}_{a b}\right. \\
& \left.-r_{0}^{2} \cos ^{2} \theta \sinh ^{2} \alpha \gamma_{a b}\right],
\end{aligned}
$$

and the charge currents

$$
\begin{aligned}
J_{2} & =\mathcal{C} r_{0}^{2} \sinh ^{2} \alpha \sin \theta \cos \theta v \wedge w, \\
J_{4} & =\mathcal{C} r_{0}^{2} \sinh \alpha \cosh \alpha \sin \theta *(v \wedge w), \\
\dot{\mathrm{I}}_{6} & =\mathcal{C} r_{0}^{2} \sinh \alpha \cosh \alpha \cos \theta * 1,
\end{aligned}
$$

where $\mathcal{C}=\left[1 /(2 \pi)^{5} g_{s}^{2} \ell_{s}^{8}\right]$ and $\gamma_{a b}$ is the induced metric on the five brane world volume (parametrized by $\sigma^{a}$ with 
$a, b, \ldots=0,1, \ldots, 5)$. The Hodge dual of $\gamma_{a b}$ is $*$ and $\hat{h}_{a b}$ is a projector onto the directions of the dissolved $\overline{D 3}$-brane charge inside the five brane. General boosts or rotations of the D3-NS5 bound state solution are expressed in terms of the velocity timelike unit vector $u^{a}$ and the spacelike orthonormal vectors $v^{a}, w^{a}$. In terms of these vectors $\hat{h}_{a b}=\gamma_{a b}-v_{a} v_{b}-w_{a} w_{b}$. The electric currents $J_{4}, \dot{\mathrm{j}}_{6}$ express the D3, NS5 currents of the solution while $J_{2}$ is a consequence of the nonzero $C_{2}$ field of the solution. $r_{0}$ is the Schwarzschild radius. In the extremal limit, $r_{0} \rightarrow 0$, $\alpha \rightarrow \infty$ while the combination $r_{0}^{2} e^{2 \alpha}$ is kept fixed. The parameter $\theta$ controls how much $\overline{D 3}$ brane charge is dissolved inside the NS5 brane.

The general effective blackfold equations of the $\overline{D 3}$-NS5 brane in the presence of 3-form NSNS/RR fluxes, constant dilaton and vanishing 1-, 5-form RR fluxes are [20]

$$
\begin{aligned}
& \nabla_{a} T^{a \mu}= \frac{g_{s}^{-1}}{6 !} H_{7}^{\mu a_{1} \cdots a_{6}} \dot{\mathrm{I}}_{6 a_{1} \cdots a_{6}}+\frac{1}{2 !} F_{3}^{\mu a_{1} a_{2}} J_{2 a_{1} a_{2}} \\
&+\frac{3}{4 !} H_{3}^{\mu a_{1} a_{2}} C_{2}^{a_{3} a_{4}} J_{4 a_{1} \cdots a_{4}}, \\
& d \star J_{2}+H_{3} \wedge \star J_{4}=0, \\
& d \star J_{4}-\star \dot{\mathfrak{l}}_{6} \wedge F_{3}=0, \quad d \star \dot{\mathfrak{l}}_{6}=0 .
\end{aligned}
$$

Note that the index $\mu$ in Eq. (7) is an index in the tendimensional ambient KS background metric. Similarly, $\star$ in Eqs. (8), (9) is the Hodge dual for the $10 d$ KS metric.

The differential equations (7)-(9) should be viewed as a system of dynamical equations for the unknown degrees of freedom of a $6 d$ effective theory. These degrees of freedom include the transverse scalars (that express $\gamma_{a b}$ ), the functions $r_{0}, \alpha, \theta$ and the orthonormal vectors $u^{a}, v^{a}, w^{a}$.

The last equation in (9) is equivalent to the statement that the NS5-brane charge density $Q_{5}=\mathcal{C} r_{0}^{2} \sinh \alpha \cosh \alpha \cos \theta$ is a constant of motion proportional to the number $N_{5}$ of NS5 branes. For five brane configurations wrapping an $S^{2}$ inside the $S^{3}$ in Eq. (1), as considered below, the first equation in Eq. (9) similarly implies that the 3-brane Page charge $Q_{3}=\int_{S^{2}} *\left[J_{4}+\star\left(\star \dot{\mathrm{j}}_{6} \wedge C_{2}\right)\right]$ is also a constant of motion proportional to the induced $D 3$-brane charge of the bound state. We denote $Q_{3} /\left(4 \pi Q_{5}\right)=\pi p g_{s} \ell_{s}^{2}$, using $p$ from now on as the ratio of the number of anti-D3s to NS5s.

Recovering KPV at extremality.-Consider the extremal limit of the Eqs. (7)-(9). We focus on a possibly timedependent configuration where the five brane bound state at $r=0$ wraps an $S^{2}$ inside the $S^{3}$ of the KS background. Writing the $S^{3}$ metric as $d \Omega_{3}^{2}=d \psi^{2}+$ $\sin ^{2} \psi\left(d \omega^{2}+\sin ^{2} \omega d \varphi^{2}\right)$ we turn on the single transverse scalar $\psi=\psi(t)$ in static gauge $\left(t=\sigma^{0}, x^{i}=\sigma^{i}\right.$, $\left.\omega=\sigma^{4}, \varphi=\sigma^{5}, i=1,2,3\right)$ and set $v^{a} \partial_{a}=\left(\sqrt{g_{s} M} \ell_{s} b_{0}\right.$ $\sin \psi)^{-1} \partial_{\omega}, w^{a} \partial_{a}=\left(\sqrt{g_{s} M} \ell_{s} b_{0} \sin \psi \sin \omega\right)^{-1} \partial_{\varphi}, u^{a} \partial_{a}=$ $\left(\sqrt{g_{s} M} \ell_{s} b_{0} \sqrt{1-\psi^{\prime 2}}\right)^{-1} \partial_{t}$. Then, the full set of Eqs. (7)-(9) reduces to

$$
\begin{aligned}
\tan \theta= & \frac{1}{b_{0}^{2} \sin ^{2} \psi}\left(\frac{\pi p}{M}-\psi+\frac{1}{2} \sin (2 \psi)\right), \\
\cot \psi= & \frac{1}{b_{0}^{2}} \sqrt{1-\psi^{\prime 2}} \sqrt{1+\tan ^{2} \theta}+\frac{1}{b_{0}^{2}} \tan \theta \\
& -\frac{1}{2}\left(1+\tan ^{2} \theta\right) \frac{\psi^{\prime \prime}}{1-\psi^{\prime 2}} .
\end{aligned}
$$

After eliminating $\tan \theta$, it is trivial to check that the resulting equation for $\psi$ coincides with the Euler-Lagrange equations of the DBI action obtained in Ref. [2] by $S$ duality of the $D 5$ brane. In this manner, we recover the equations of motion of the KPV effective action from supergravity. A more general relation between the extremal blackfold equations and DBI equations can be derived along the lines of Ref. [24] (see also Ref. [25]).

The maximum value $p^{*}$ that allows a metastable vacuum has the following meaning in the blackfold language. Since the NS5 branes at extremality have a nonvanishing Hagedorn local temperature $T_{H}=(1 / 2 \pi) \sqrt{\left(\mathcal{C} / Q_{5}\right)} \sqrt{|\cos \theta|}$, one can show that $p^{*}$ is very close to the point $\hat{p}$ where this temperature takes its maximum possible value. The latter occurs when $\cos \hat{\theta}=1$, i.e. when the 3 -brane charge is depleted. From Eq. (11) we deduce that at that point $\hat{\psi} \simeq$ 0.7506 obeying the equation $\cot \hat{\psi}=\frac{1}{b_{0}^{2}}$. Then, Eq. (10) gives $\frac{\hat{p}}{M}=\hat{\psi}-\frac{1}{2} \sin (2 \hat{\psi}) \simeq 0.08$, also noted in Ref. [2].

Nonextremal static configurations. - It is straightforward to repeat the above exercise for non-extremal configurations at finite $\alpha$. We continue to focus on the same ansatz for the world volume fields, now restricting to timeindependent profiles. The nonextremal, static version of Eq. (10) is exactly the same as before. On the other hand, when expressed in terms of $\alpha$ Eq. (11) becomes

$\cot \psi=\frac{1}{b_{0}^{2}}\left(\frac{\operatorname{coth} \alpha}{\cos \theta}+\tan \theta\right) \frac{2 \cos ^{2} \theta}{2 \cos ^{2} \theta+(\sinh \alpha)^{-2}}$.

This equation can be written alternatively in terms of the local temperature $T=\left(1 / 2 \pi r_{0} \cosh \alpha\right)$, or the local entropy density $s=2 \pi \mathcal{C} r_{0}^{3} \cosh \alpha$, or any other quantity that characterises the deviation from extremality. We refer the reader to Sec. I in Supplemental Material [26] for a derivation of the equations of motion from an effective potential (using in part results of Refs. [27,28]). In what follows, we will make use of an effective potential $V_{S}$ that keeps the entropy $S$ fixed.

Results for nonextremal configurations.-Consider first the regime $p / M<p^{*} / M \simeq 0.08$, where the extremal solutions have a meta-stable vacuum. In Fig. 1 we show how the effective potential $V_{S}$ changes as we vary the entropy $S$ for a fixed value of $p / M$. We observe two interesting new features. First, as soon as $S$ is turned on, a new unstable vacuum emerges (black dots on the right plot of Fig. 1) near the North pole, $\psi \simeq 0$. For sufficiently small 

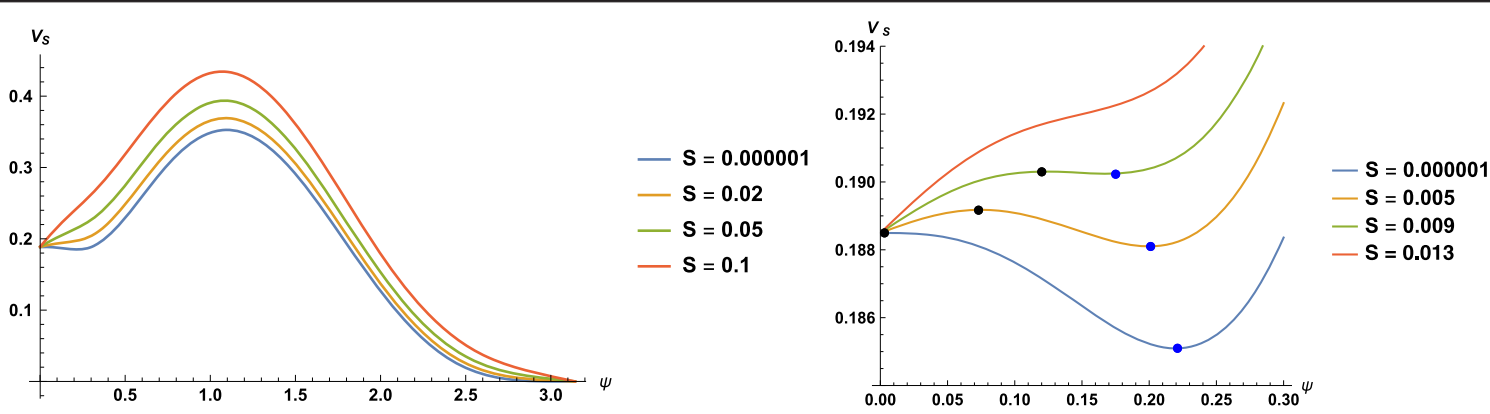

FIG. 1. Plots of the effective potential at fixed entropy $V_{S}$, as a function of the angle $\psi$ on $S^{3}$. Both figures represent plots at $p / M=0.03$. The right plot zooms into the region near the North pole of the $S^{3}$. As we increase the entropy we encounter a critical value $S^{*}$, where the metastable vacuum of KPV (blue dots on the right) merges with a new unstable vacuum (black dots on the left).

values of $S$ there are three extrema: two unstable and one metastable. Second, as we increase $S$ further the new unstable extremum comes closer to the metastable vacuum and the two merge at a critical value of the entropy $S^{*}$, which is a function of $p / M$. Above this value the metastable vacuum is lost. The new unstable state represents a fat black NS5 with a highly pinched $\mathbb{R}^{3} \times S^{5}$ horizon geometry that resembles a black $\overline{D 3}$. Instead, the metastable state starts life near extremality as a thin black NS5 with $\mathbb{R}^{3} \times S^{2} \times S^{3}$ horizon topology. At the merger the metastable black NS5 turns effectively into a black $\overline{D 3}$. The picture of a merger driven by horizon geometry is reinforced by the following observation.

A quantitative measure of the "fatness" of a black NS5 wrapping an $S^{2}$ is provided by the ratio $d=2 \sqrt{p} \hat{r}_{0} /$ $[\sqrt{M} \sin (\psi)]$ [29], where $\hat{r}_{0} \equiv \sqrt{\mathcal{C}} r_{0} / \sqrt{Q_{5}}$ is dimensionless. The ratio $d$, which is a natural function of $p / M$ and the equilibrium $\psi$, compares the scale $2 \sqrt{g_{s} p} \hat{r}_{0} \ell_{s}=$ $2\left(g_{s} p N_{5}^{-1}\right)^{1 / 2} r_{0}$ associated to the Schwarzschild radius and the scale of the $S^{2}$ wrapped by the NS5 world volume $\sqrt{g_{s} M} \ell_{s} \sin (\psi)$. As an illustration, on the left plot in Fig. 2 we see how $d$ behaves in the unstable branch (blue color) and the metastable branch (orange color). Recall that $\alpha \rightarrow \infty$ represents extremality. The unstable branch has visibly higher values of $d$, expressing the dominance of the Schwarzschild radius. The metastable branch captures a thin black NS5 with small values of $d$. The merger occurs at a value of $d$ notably close to 1 .

On the right plot of Fig. 2 we show how $d$ at the merger point behaves as a function of $p / M$. Remarkably, the ratio remains effectively constant, near the value 0.89 over a significant range. It deviates slightly from this value in the vicinity of the upper bound of $p / M$, where effects from the second unstable state (already visible in KPV [2]) become important. The characteristically weak dependence of $d$ on $p / M$ is a clear signal that the properties of the merger point are closely tied to the properties of the horizon geometry. Finally, by increasing $p / M$ further, above the critical value $p^{*} / M \simeq 0.08$, we observe the complete loss of the metastable vacuum exactly as in the extremal KPV analysis [2]. The unstable vacuum in the vicinity of the North pole, however, remains, even above $p^{*} / M$, and constitutes the single vacuum of the nonextremal static blackfold equations.

Discussion.-The results of the blackfold analysis are consistent in the regime of large $g_{s} p$ when $N_{5} \ll g_{s} M \sin ^{2} \psi$ and $\sqrt{p / M} \ll \sqrt{g_{s} M} \sin ^{2} \psi$ (see Sec. II of Supplemental Material [26]). For sufficiently large $M$ our calculations are therefore valid everywhere except for a small region around the North pole. At extremality we recover the results of KPV [2] in a regime very different from theirs $[p \sim \mathcal{O}(1)]$. Moving away from extremality was so far impossible. Our analysis reveals two novel features of black $\overline{D 3}$-NS5 branes: a new unstable state near the North pole, and the
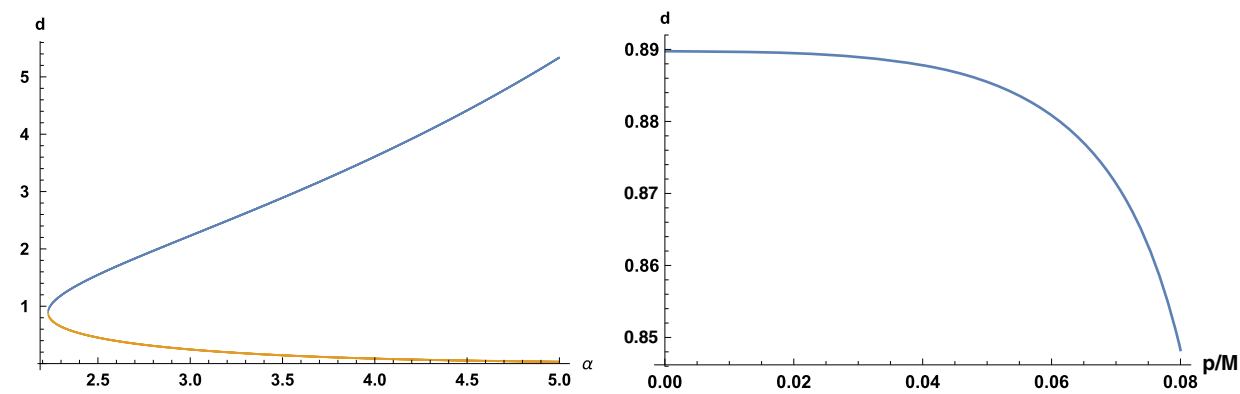

FIG. 2. Plots of the ratio $d$ that expresses how "fat" a nonextremal $\overline{D 3}$-NS5 bound state is. On the left plot we depict the dependence of $d$ on the nonextremality parameter $\alpha$ for the unstable (blue) and metastable (orange) branches for $p / M=0.03$. On the right plot, we depict $d$ at the critical merger point as a function of $p / M$. 
persistence of the metastable state for small enough horizon radius. For a critical value of the horizon radius the metastable state and the new unstable state merge. Above this critical value, the metastable state is lost. Remarkably, these two features coincide with what was anticipated in Ref. [10] based on a very complementary viewpoint that extracted features from would-be exact solutions of NS5 branes. A nogo-theorem, based on singularities, implies that the black NS5, if it exists, has a maximum horizon radius until it disappears. A black $\overline{D 3}$ also escapes the nogo, but was deemed unphysical in Ref. [10], since it does not persist in the extremal limit. We have now shown that when the nogo's of Refs. $[10,11]$ do not apply we find go's. Together with the consistency of the extremal limit, this constitutes strong evidence for the existence of the metastable states, since they have now been argued for in complementary regimes. Our analysis should impact the understanding of finite temperature effects in confining cascading gauge theories. We leave a study of this aspect to future work.

We thank F. F. Gautason and J.P. van der Schaar for useful discussions. The work of T. V. R. is supported by the FWO odysseus Grant No. G.0.E52.14N and the C16/16/ 005 grant of the KULeuven. We acknowledge support from the European Science Foundation HoloGrav Network. The work of V. N. is supported by STFC under the consolidated Grant No. ST/P000371/1. The work of N. O. is supported in part by a grant of the Independent Research Fund Denmark (Grant No. DFF-6108-00340). J. A. is partly supported by the Netherlands Organisation for Scientific Research (NWO).

[1] J. M. Maldacena and H. S. Nastase, The Supergravity dual of a theory with dynamical supersymmetry breaking, J. High Energy Phys. 09 (2001) 024.

[2] S. Kachru, J. Pearson, and H. L. Verlinde, Brane / flux annihilation and the string dual of a nonsupersymmetric field theory, J. High Energy Phys. 06 (2002) 021.

[3] S. Kachru, R. Kallosh, A. D. Linde, and S. P. Trivedi, De Sitter vacua in string theory, Phys. Rev. D 68, 046005 (2003).

[4] S. Kachru, R. Kallosh, A. D. Linde, J. M. Maldacena, L. P. McAllister, and S. Trivedi, Towards inflation in string theory, J. Cosmol. Astropart. Phys. 10 (2003) 013.

[5] I. Bena, A. Puhm, and B. Vercnocke, Non-extremal black hole microstates: Fuzzballs of fire or fuzzballs of fuzz, J. High Energy Phys. 12 (2012) 014.

[6] I. Bena, M. Graña, and N. Halmagyi, On the existence of meta-stable vacua in Klebanov-Strassler, J. High Energy Phys. 09 (2010) 087.

[7] F. F. Gautason, D. Junghans, and M. Zagermann, Cosmological constant, near brane behavior and singularities, J. High Energy Phys. 09 (2013) 123.
[8] J. Blåbäck, U. H. Danielsson, and T. Van Riet, Resolving anti-brane singularities through time-dependence, J. High Energy Phys. 02 (2013) 061.

[9] B. Michel, E. Mintun, J. Polchinski, A. Puhm, and P. Saad, Remarks on brane and antibrane dynamics, J. High Energy Phys. 09 (2015) 021.

[10] D. Cohen-Maldonado, J. Diaz, T. Van Riet, and B. Vercnocke, Observations on fluxes near anti-branes, J. High Energy Phys. 01 (2016) 126.

[11] D. Cohen-Maldonado, J. Diaz, and F. F. Gautason, Polarised antibranes from Smarr relations, J. High Energy Phys. 05 (2016) 175.

[12] I. Bena, A. Buchel, and O. J. C. Dias, Horizons cannot save the landscape, Phys. Rev. D 87, 063012 (2013).

[13] I. Bena, J. Blåbäck, U. H. Danielsson, and T. Van Riet, Antibranes cannot become black, Phys. Rev. D 87, 104023 (2013).

[14] J. Blåbäck, U. H. Danielsson, D. Junghans, T. Van Riet, and S. C. Vargas, Localised anti-branes in non-compact throats at zero and finite $T$, J. High Energy Phys. 02 (2015) 018.

[15] G. S. Hartnett, Localised anti-branes in flux backgrounds, J. High Energy Phys. 06 (2015) 007.

[16] S. S. Gubser, Curvature singularities: The Good, the bad, and the naked, Adv. Theor. Math. Phys. 4, 679 (2000).

[17] I. Bena, M. Graa, S. Kuperstein, and S. Massai, Giant tachyons in the landscape, J. High Energy Phys. 02 (2015) 146.

[18] R. Emparan, T. Harmark, V. Niarchos, and N. A. Obers, World-Volume Effective Theory for Higher-Dimensional Black Holes, Phys. Rev. Lett. 102, 191301 (2009).

[19] R. Emparan, T. Harmark, V. Niarchos, and N. A. Obers, Essentials of Blackfold dynamics, J. High Energy Phys. 03 (2010) 063.

[20] J. Armas, J. Gath, V. Niarchos, N. A. Obers, and A. V. Pedersen, Forced fluid dynamics from Blackfolds in general supergravity backgrounds, J. High Energy Phys. 10 (2016) 154.

[21] I. R. Klebanov and M. J. Strassler, Supergravity and a confining gauge theory: Duality cascades and chi SB resolution of naked singularities, J. High Energy Phys. 08 (2000) 052.

[22] M. Alishahiha, Y. Oz, and M. M. Sheikh-Jabbari, Supergravity and large N noncommutative field theories, J. High Energy Phys. 11 (1999) 007.

[23] T. Harmark and N. A. Obers, Phase structure of noncommutative field theories and spinning brane bound states, J. High Energy Phys. 03 (2000) 024.

[24] V. Niarchos, Open/closed string duality and relativistic fluids, Phys. Rev. D 94, 026009 (2016).

[25] G. Grignani, T. Harmark, A. Marini, and M. Orselli, Born-infeld/gravity correspondence, Phys. Rev. D 94, 066009 (2016).

[26] See Supplemental Material at http://link.aps.org/ supplemental/10.1103/PhysRevLett.122.181601 for details on nonextremal thermodynamic potentials and regimes of validity.

[27] R. Emparan, T. Harmark, V. Niarchos, and N. A. Obers, Blackfolds in supergravity and string theory, J. High Energy Phys. 08 (2011) 154. 
[28] J. Armas, J. Gath, A. Jain, and A. V. Pedersen, Dissipative hydrodynamics with higher-form symmetry, J. High Energy Phys. 05 (2018) 192.

[29] Our definition of $d$ contains a rescaling of $r_{0}$ in such a way that we recover the known anti-D3 energy in the extremal limit, given by $2 p g_{s}^{-1}$, where we work in conventions for which the warp factor at the tip has been absorbed in a rescaling of the Minkowskian coordinates. That explains, in particular, the factor of 2 in the definition of $d$. 